T. Ohsawa

Nagoya Math. J.

Vol. 141 (1996), 143-156

\title{
ON THE ANALYTIC STRUCTURE OF CERTAIN INFINITE DIMENSIONAL TEICMÜLLER SPACES
}

\author{
TAKEO OHSAWA
}

\section{Introduction}

It is well known since long time that quasiconformally different finite Riemann surfaces give rise to biholomorphically nonequivalent Teichmüller spaces except for a few obvious cases (cf. [R], [E-K]). This is deduced as an application of Royden's theorem asserting that the Teichmüller metric is equal to the Kobayashi metric. For the case of infinite Riemann surfaces, however, it is still unknown whether or not the corresponding result holds, although it has been shown by $\mathrm{F}$. Gardiner $[G]$ that Royden's theorem is also valid for the infinite dimensional Teichmüller spaces. On the other hand, recent activity of several mathematicians shows that the infinite dimensional Teichmüller spaces are interesting objects of complex analytic geometry (cf. $[\mathrm{Kru}],[\mathrm{T}],[\mathrm{N}],[\mathrm{E}-\mathrm{K}-\mathrm{K}]$ ). Therefore, based on the generalized form of Royden's theorem, one might well look for further insight into Teichmüller spaces by studying the above mentioned nonequivalence question.

In the present article, we shall restrict our attention to the connected sum of infinitely many finite Riemann surfaces and show that, if their necks are sufficiently long at infinity, one can naturally associate to each quasiconformal equivalence class of such surfaces the cofinal equivalence class of a sequence of nonnegative integers which distinguishes the infinitesimal forms of the Teichmüller metric (cf. Proposition 3.3). For that we shall show in section one, that the space of integr able holomorphic quadratic differentials decomposes asymptotically as the surface is pinched along a simple closed geodesic to two hyperbolic surfaces (cf. Theorem 1.5). The proof of this fact relies on the method of solving the $\bar{\partial}$-equation with $L^{2}$ estimates as developed by $[\mathrm{A}-\mathrm{V}],[\mathrm{O}-1,2]$ and $[\mathrm{D}]$ on complete Kähler manifolds. This, combined with an elementary argument on the quasi-isometric equivalence of normed vector spaces, allows us to distinguish the infinitesimal forms of the Teichmüller metric by the direct summands at infinity of the space of integable

Received September 5, 1994. 
quadratic differentials. As a consequence we shall conclude that there exist uncountably many nonequivalent Teichmüller spaces.

As a related result we must mention a very recent work of $C$. Earle and F. Gardiner $[\mathrm{E}-\mathrm{G}]$ that provides the distinction of Teichmüller infinitesimal forms in the case of topologically finite Riemann surfaces.

The author thanks to H. Tanigawa, J. Noguchi and S. Mukai for stimulating conversations. He also thanks to M. Taniguchi, C. Earle and J. Bland for useful comments.

\section{\$1. Asymptotic decomposition theorem}

Let $R$ be a (connected) Riemann surface. In what follows we assume that $R$ is hyperbolic, i.e. it admits the unit disc $\Delta=\{z \in \mathbf{C}|| z \mid<1\}$ as the universal covering space. We shall denote by $\pi_{R}$ a covering map $\Delta \rightarrow R$. By $d \sigma_{R}^{2}$ we denote a Hermitian metric on $R$ uniquely determined by the equation

$$
\pi_{R}^{*} d \sigma_{R}^{2}=\frac{d z d \bar{z}}{\left(1-|z|^{2}\right)^{2}} .
$$

We shall denote by $d \mu_{R}$ the volume form of $R$ with respect to $d \sigma_{R}^{2}$. Given any section $\omega$ of the multi-canonical bundle $K_{R}^{\otimes m}(m \in \mathbf{Z}),|\omega|_{R}$ will denote the (pointwise) length of $\omega$ with respect to the fiber metric of $K_{R}^{\otimes m}$ induced from $d \sigma_{R}^{2}$. For any continuous function $\phi: R \rightarrow \mathbf{R}$ and for any $p \geqq 1, L_{m}^{p}(R, \phi)$ will stand for the Banach space of measurable sections $u$ of $K_{R}^{m}$ satisfying

$$
\int_{R} e^{-\phi}|u|_{R}^{p} d \mu_{R}<\infty
$$

We put

$$
\|u\|_{p, \phi}=\left(\int_{R} e^{-\phi}|u|_{R}^{p} d \mu_{R}\right)^{1 / p}
$$

for any $u \in L_{m}^{p}(R, \phi)$. We shall not refer to $\phi$ if $\phi=0$. In the later argument we shall use those $\phi$ for which $\pi^{*} \phi(z)-\log \left(1-|z|^{2}\right)$ is a subharmonic function on $\Delta$. By the symbol $\bar{\partial}$ we mean the complex exterior derivative of type $(0,1)$ acting on $L_{m}^{p}(R, \phi)$ in the sense of distribution. Our main concern is to know how geometry of $R$ is embodied in the Banach space structure of $A_{m}^{p}(R):=L_{m}^{p}(R) \cap$ $\operatorname{Ker} \bar{\partial}$. Applications to Teichmüller spaces in mind, we shall only study $A_{2}^{1}(R)$ below, although we shall need certain information on $A_{2}^{2}(R, \phi)$ by a tech- 
nical reason.

First let us consider the annuli

$$
\Delta\left(r_{1}, r_{2}\right):=\left\{z \in \mathbf{C}\left|r_{1}<\right| z \mid<r_{2}\right\} \quad\left(0 \leqq r_{1}<r_{2} \leqq \infty\right) .
$$

For any $\omega \in A_{2}^{1}\left(\Delta\left(r_{1}, r_{2}\right)\right)$ we consider its Laurent expansion

$$
\omega(z)=\left(\sum_{k \in Z} c_{k} z^{k}\right) d z^{\otimes 2}
$$

and put

$$
\begin{aligned}
& \omega_{+}(z)=\left(\sum_{k>-2} c_{k} z^{k}\right) d z^{\otimes 2} \\
& \omega_{-}(z)=\left(\sum_{k<-2} c_{k} z^{k}\right) d z^{\otimes 2} \\
& \omega_{0}(z)=c_{-2} z^{-2} d z^{\otimes 2}
\end{aligned}
$$

Without loosing generality we may assume that $r_{1}=r_{2}^{-1}$, since

$$
\Delta\left(r_{1}, r_{2}\right) \simeq \Delta\left(\sqrt{r_{1} / r_{2}}, \sqrt{r_{2} / r_{1}}\right) .
$$

Lemma 1.1. There exists a constant $C$ such that, for any $\varepsilon \in(0,1)$ and for any $r \in\left(0, \frac{1}{2}\right)$ the following inequalities hold for all $\omega \in A_{2}^{1}\left(\Delta\left(r, r^{-1}\right)\right)$.

$$
\begin{gathered}
\int_{\Delta\left(0, r^{\varepsilon-1}\right)}\left|\omega_{+}\right| \leq C r^{\varepsilon}\|\omega\|_{1} \\
\int_{\Delta\left(r^{1-\varepsilon}, \infty\right)}\left|\omega_{-}\right| \leq C r^{\varepsilon}\|\omega\|_{1} \\
\int_{\Delta\left(r, r^{1-\varepsilon}\right) \cup \Delta\left(r^{-1+\varepsilon}, r^{-1}\right)}\left|\omega_{0}\right| \leq C \varepsilon\|\omega\|_{1}
\end{gathered}
$$

Proof. Let $\theta_{r}: \Delta\left(r^{2}, 1\right) \rightarrow \Delta\left(r, r^{-1}\right)$ be a biholomorpic map given by $\theta_{r}(z)=z / r$. Then

$$
\|\omega\|_{1}=\int_{\Delta\left(r^{2}, 1\right)}\left|\theta_{r}^{*} \omega\right|
$$

and

$$
\int_{\Delta\left(0, r^{\varepsilon-1}\right)}\left|\omega_{+}\right|=\int_{\Delta\left(0, r^{\varepsilon}\right)}\left|\theta_{r}^{*} \omega_{+}\right|
$$

From the assumption $\Delta\left(\frac{1}{4}, 1\right) \subset \Delta\left(r^{2}, 1\right)$. Hence 


$$
\int_{\Delta(1 / 3,1 / 2)}\left|\theta_{r}^{*} \omega\right|_{\Delta}^{2} d u_{\Delta} \leq C_{1}\|\omega\|_{1}^{2}
$$

for some constant $C_{1}$, since the $L^{p}$-norms are all locally equivalent for holomorphic functions.

Clearly

$$
\int_{\Delta(1 / 3,1 / 2)}\left|\theta_{r}^{*} \omega_{+}\right|^{2} d u_{\Delta} \leq \int_{\Delta(1 / 3,1 / 2)}\left|\theta_{r}^{*} \omega\right|_{\Delta}^{2} d u_{\Delta}
$$

Moreover, by Cauchy's inequality and the maximum principle, there exists a constant $C_{2}$ such that, for any holomorphic function $f$ on $\Delta(0,1)$ with a pole of order at most one at 0 , the inequality

$$
|f(z)|^{2} \leq C_{2}|z|^{-2} \int_{\Delta(1 / 3,1 / 2)}|f(\zeta)|^{2} d \mu_{\Delta}(\zeta)
$$

holds for any $z \in \Delta\left(0, \frac{1}{3}\right)$.

Combining (1.4) through (1.7) one immediately gets (1.1). The inequality (1.2) is reduced to (1.1) by the inversion $z \rightarrow z^{-1}$.

In view of (1.1) and (1.2), (1.3) is a consequence of the equality

$$
\frac{\int_{r}^{r^{1-\varepsilon}} \frac{d t}{t}+\int_{r^{\varepsilon-1}}^{r^{-1}} \frac{d t}{t}}{\int_{r^{1-\varepsilon}}^{r^{\varepsilon-1}} \frac{d t}{t}}=\frac{\varepsilon}{1-\varepsilon} .
$$

For simplicity we set

$$
J S(r)=\mathbf{C} \cdot z^{-2} d z^{\otimes 2} \subset A_{2}^{1}\left(\Delta\left(r, r^{-1}\right)\right) \text { for any } r>0
$$

and define a bijective linear map $T_{r}$ from $A_{2}^{1}\left(\Delta\left(r, r^{-1}\right)\right)$ to $A_{2}^{1}\left(\Delta\left(0, r^{-1}\right)\right) \oplus$ $A_{2}^{1}(\Delta(r, \infty)) \oplus J S(r)$ by $T_{r}(\omega)=\left(\omega_{+}, \omega_{-}, \omega_{0}\right)$. Then as an immediate consequence of Lemma 1.1, we obtain the following decomposition theorem.

TheOREm 1.2. For any $\varepsilon>0$ there exists an $r>0$ such that

$$
(1-\varepsilon)\|\omega\|_{1} \leqq\left\|T_{r}(\omega)\right\|_{1} \leqq(1+\varepsilon)\|\omega\|_{1}
$$

for all $\omega \in A_{2}^{1}\left(\Delta\left(r, r^{-1}\right)\right)$. Here the $L^{1}$-norm \|\|$_{1}$ of $T_{r}(\omega)$ is defined as the sum of those of the components. 
We shall generalize Theorem 1.2 from the annuli to those Riemann surfaces that are pinchable to two hyperbolic surfaces. For that we first recall the following (cf. [O-2] or [D]).

THEOREM 1.3. Let $M$ be a complex manifold of dimension $n$ that admits a complete Kähler metric and let $(E, h)$ be a positive Hermitian line bundle over $M$. Then, for any $q>0$ and any $\bar{\partial}$-closed $(n, q)$-form $v$ on $M$ which is square integrable with respect to $\theta=\sqrt{-1} \partial \partial \partial \log h$ and $h$, there exists an $(n, q-1)$-form $u$ on $M$ such that $\bar{\partial} u=v$ and $\|u\|_{h, \theta} \leqq\|v\|_{h, \theta}$. Here \|\|$_{h, \theta}$ denotes the $L^{2}$-norm with respect to $h$ and $\theta$.

Applying this to Riemann surfaces we shall prove a lemma which generalizes the property (1.1) of the correspondence $\omega \rightarrow \omega_{+}$.

Let $R$ be any hyperbolic Riemann surface of the form $\hat{R} \backslash\{p\}$ for some Riemann surface $\hat{R}$ and $p \in \hat{R}$, and let $z$ be a local coordinate around $p$ which maps a neighbourhood $D$ of $p$ biholomorphically onto the unit disc. We put $D_{r}:=$ $\{q \in D|| z(q) \mid<r\}$ for $r \in(0,1)$. By $\rho_{r}$ we shall denote the restriction map from $A_{2}^{1}(R)$ to $A_{2}^{1}\left(\hat{R} \backslash \bar{D}_{r}\right)$.

LEMma 1.4. For any $r \in\left(0, \frac{1}{9}\right)$ one can find a continuous linear map $\phi_{r}$ from $A_{2}^{1}\left(\hat{R} \backslash \bar{D}_{r}\right)$ to $A_{2}^{1}(R)$ so that $\left\{\phi_{r}\right\}_{r \in(0,1 / 9)}$ satisfies the following.

$$
\phi_{r}^{\circ} \rho_{r}=\mathrm{id} \text { for all } r \text {. }
$$

(1.10) There exists a constant $C_{3}=C_{3}(R, D, p)$ such that for all $\varepsilon \in\left(0, \frac{1}{2}\right)$ with $\varepsilon \log r<-\log 3$,

$$
\begin{aligned}
& \int_{\widehat{R}_{\widehat{D}} \bar{D}_{r} \varepsilon}\left|\phi_{r}(\omega)-\omega\right|+\int_{\left.D_{r} \varepsilon \backslash p\right\}}\left|\phi_{r}(\omega)\right| \leqq C_{3} \varepsilon\|\omega\|_{1} \\
& \text { holds for all } \omega \in A_{2}^{1}\left(\hat{R} \backslash \bar{D}_{r}\right) \text {. }
\end{aligned}
$$

Proof. We fix any point $p^{\prime}$ on $\partial D_{1 / 2}$ and a covering map $\pi$ from $\Delta$ onto $R$ satisfying $\pi(0)=p^{\prime}$. Then we define a real-valued continuous function $\phi$ on $R$ by

$$
\phi(q)=\sup _{z \in \pi^{-1}(q)} \log \left(1-|z|^{2}\right) .
$$

Clearly $\phi$ is continuous and enjoys the following two properties. 
(1.11) There exists a constant $C_{4}$ such that

$C_{4}^{-1} \phi(q) \leq \operatorname{dist}\left(p^{\prime}, q\right) \leqq C_{4} \phi(q)$ for any $q \in R$, where $\operatorname{dist}\left(p^{\prime}, q\right)$ denotes the distance between $p^{\prime}$ and $q$ with respect to $d \sigma_{R}^{2}$.

$$
\sqrt{-1}\left(\partial \bar{\partial} \pi^{*} \phi+\frac{d z \wedge d \bar{z}}{\left(1-|z|^{2}\right)^{2}}\right) \geqq 0 .
$$

As a consequence of (1.11) we have

$$
\int_{R} e^{(1+\eta) \phi} d \mu_{R}<\infty \quad \text { for any } \eta>0
$$

Let $\chi: \mathbf{R} \rightarrow \mathbf{R}$ be any $C^{\infty}$ function satisfying $\chi \mid(-\infty, 1 / 3) \equiv 0$ and $\chi \mid(1 / 2, \infty)$ $\equiv 1$. Then we define $\phi_{r}$ as follows.

Given any $\omega \in A_{2}^{1}\left(\hat{R} \backslash \bar{D}_{r}\right)$ we define a $K_{R}^{\otimes 2}$-valued $(0,1)$-form $v$ on $R$ by

$$
v= \begin{cases}\left(\omega-\left(\omega \mid D \backslash \bar{D}_{r}\right)_{+}\right) \bar{\partial} \chi(|z|) & \text { on } D \backslash D_{1 / 3} \\ 0 & \text { otherwise. }\end{cases}
$$

Since the pointwise norm of $\bar{\partial} \chi(|z|)$ is bounded by const $\operatorname{dist}\left(\partial D_{1}, \partial D_{1 / 3}\right)$, applying Lemma 1.1 to estimate the norm of $\omega-\left(\omega \mid D \backslash \bar{D}_{r}\right)_{+}$from above, we obtain by a straightforward application of the Cauchy-Schwartz inequality,

$$
\sup _{\omega \neq 0} \frac{\|v\|_{2,3 \phi / 2}}{\|\omega\|_{1}} \leqq C_{5}|\log r|^{-1}
$$

for some constant $C_{5}$ which depends only on $\operatorname{dist}\left(\partial D_{1}, \partial D_{1 / 3}\right)$.

Regarding $v$ as a $K_{R}$-valued (1,1)-form on $R$ and applying Theorem 1.3 for $M=R$ by utilizing the curvature inequality (1.12) with respect to the regularizations of $\phi$ that approximate $\phi$ from above (cf. [Ri]) we obtain a section $u$ of $K_{R}^{\otimes 2}$ over $R$ satisfying

$$
\left\{\begin{array}{l}
\bar{\partial} u=v \\
u \perp \operatorname{Ker} \bar{\partial} \\
\|u\|_{2,3 \phi / 2}^{2} \leqq 2\|v\|_{2,3 \phi / 2}^{2} .
\end{array}\right.
$$

Then we put

$$
\phi_{r}(\omega)=\left\{\begin{array}{l}
\left(\omega \mid D \backslash \bar{D}_{r}\right)_{+}+\chi(|z|)\left(\omega-\left(\omega \mid D \backslash \bar{D}_{r}\right)_{+}\right)-u \text { on } D \backslash\{p\} \\
\omega+(\chi(|z|)-1)\left(\omega-\left(\omega \mid D \backslash \bar{D}_{r}\right)_{+}\right)-u \text { on } \hat{R} \backslash D_{1 / 3} .
\end{array}\right.
$$

The desired estimate for $\Phi_{r}(\omega)-\omega$ and $\Phi_{r}(\omega)$ follows directly from (1.2), (1.3), (1.14), (1.15) and 


$$
\int_{R}|u| \leqq\|u\|_{2,3 \phi / 2}\left(\int_{R} e^{3 \phi / 2} d \mu_{R}\right)^{1 / 2}
$$

To provide a situation under which we can generalize Theorem 1.2 , let $R_{i}(i=1,2)$ be two hyperbolic Riemann surfaces of the form $\hat{R}_{i} \backslash\left\{p_{i}\right\}$, and let $z_{i}$ : $D_{i} \rightarrow \Delta$ be local coordinates around $p_{i}$. Then for any $r \in(0,1)$ we define a Riemann surface $R[r]$ by patching $\hat{R}_{i} \backslash\left\{\left|z_{\imath}\right| \leqq r\right\}$ along $D_{i} \backslash\left\{\left|z_{i}\right| \leqq r\right\}$ via the correspondence

$$
\begin{array}{ccc}
D_{1} \backslash\left\{\left|z_{1}\right| \leqq r\right\} & \longrightarrow & D_{2} \backslash\left\{\left|z_{2}\right| \leqq r\right\} \\
\psi & & w \\
q & \longrightarrow & z_{2}^{-1}\left(r / z_{1}(q)\right) .
\end{array}
$$

We put $D_{i}(r)=\left\{q \in \hat{R}_{i}|| z_{i}(q) \mid<r\right\}$. Then the following generalizes Theorem 1.2 .

THEOREM 1.5. For any $\varepsilon>0$ there exist an $r_{0}>0$, depending on $\varepsilon$ and $\operatorname{dist}\left(\partial D_{i}(1), \partial D_{i}(1 / 3)\right)(i=1,2)$, and a bijective linear map $\mathscr{T}_{r}$ from $A_{2}^{1}(R[r])$ to $A_{2}^{1}\left(R_{1}\right) \oplus A_{2}^{1}\left(R_{2}\right) \oplus J S(r)$ satisfying

$$
(1-\varepsilon)\|\omega\|_{1} \leqq\left\|\mathscr{T}_{r}(\omega)\right\|_{1} \leqq(1+\varepsilon)\|\omega\|_{1}
$$

for all $\omega \in A_{2}^{1}(R[r])$.

Proof. Let $D_{i}(r)=\left\{q \in \hat{R}_{i}|| z_{i}(q) \mid<r\right\}$. By Lemma 1.4 there exist a constant $C_{6}$ and continuous linear maps $\Phi_{i r}$ from $A_{2}^{1}\left(\hat{R}_{i} \backslash \overline{D_{i}(r)}\right)$ onto $A_{2}^{1}\left(R_{i}\right)$ for $r \in$ $\left(0, \frac{1}{9}\right)$ such that

$$
\int_{\hat{R}_{i} \backslash \overline{D_{i}\left(r^{\varepsilon}\right)}}\left|\Phi_{i r}\left(\omega_{i}\right)-\omega_{i}\right|+\int_{D_{i}\left(r^{\varepsilon}\right) \backslash\left\{p_{i}\right\}}\left|\Phi_{i r}\left(\omega_{i}\right)\right| \leqq C_{6} \varepsilon\left\|\omega_{i}\right\|_{1}
$$

for all $\omega_{i} \in A_{2}^{1}\left(\hat{R}_{1} \backslash \overline{D_{i}(r)}\right)$ provided that $r \in(0, \exp (-(\log 3) / \varepsilon))$.

Then we put

$$
\begin{aligned}
\mathscr{T}_{r}(\omega)= & \left(\Phi_{1 r}\left(\omega \mid \hat{R}_{1} \backslash \overline{D_{1}(r)}\right), \Phi_{2 r}\left(\omega \mid \hat{R}_{2} \backslash \overline{D_{2}(r)}\right),\right. \\
& \left.\left(\left(\theta_{\sqrt{r}}^{-1} \circ z_{1}^{-1}\right)^{*}\left(\omega \mid D_{1} \backslash\left\{\left|z_{1}\right| \leqq r\right\}\right)\right)_{0}\right) .
\end{aligned}
$$

By (1.17) $\mathscr{T}_{r}$ satisfies (1.16) for sufficiently small $r$. In particular, $\mathscr{T}_{r}$ is then injective and the image of $\mathscr{T}_{r}$ is closed. Therefore the surjectivity of $\mathscr{T}_{r}$ will follow from the assertion that, for any $\left(\omega_{1}, \omega_{2}, \omega_{3}\right)$ in $A_{2}^{1}\left(R_{1}\right) \oplus A_{2}^{1}\left(R_{2}\right) \oplus \Lambda(\sqrt{r})$ there 
exists an $\omega \in A_{2}^{1}(R[r])$ such that

$$
\left\|\mathscr{T}_{r}(\omega)-\left(\omega_{1}, \omega_{2}, \omega_{3}\right)\right\|_{1} \leq \frac{1}{2}\left\|\left(\omega_{1}, \omega_{2}, \omega_{3}\right)\right\|_{1}
$$

For that one has only to repeat the argument in the proof of Lemma 1.4. The detail is thus left to the reader.

Note that the above $R[r]$ is uniquely determined by the triples $\left\{\left(R_{i}, D_{i}\right.\right.$, $\left.\left.z_{i}\right)\right\}_{i=1,2}$ and $r$. To make this dependence more explicit, we shall denote $R[r]$ by $\left(R_{1}, D_{1}, z_{1}\right) \#_{r}\left(R_{2}, D_{2}, z_{2}\right)$. We note that one may take $r_{0}=$ const $\cdot \min ($ dist $\left.\left(\partial D_{1}(1), \partial D_{1}(1 / 3)\right), \operatorname{dist}\left(\partial D_{2}(1), \partial D_{2}(1 / 3)\right) \cdot \exp (-(\log 3) / \varepsilon)\right)$.

\section{§2. Quasi-irreducible decomposition of normed vector spaces}

Let $\left(L_{i},\|\|_{(\imath)}\right)(i=1,2)$ be normed vector spaces. For any $\left(v_{1}, v_{2}\right) \in L_{1} \oplus$ $L_{2}$ we put

$$
\left\|\left(v_{1}, v_{2}\right)\right\|_{1}=\left\|v_{1}\right\|_{(1)}+\left\|v_{2}\right\|_{(2)}
$$

and

$$
\left\|\left(v_{1}, v_{2}\right)\right\|_{\infty}=\max \left\{\left\|v_{1}\right\|_{(1)},\left\|v_{2}\right\|_{(2)}\right\} .
$$

We shall call the normed vector space $\left(L_{1} \oplus L_{2},\|\|_{1}\right)$ (resp. $\left(L_{1} \oplus L_{2},\|\|_{\infty}\right)$ ) the 1 -direct sum (resp. the $\infty$-direct sum) of $\left(L_{1},\|\|_{(1)}\right)$ and $\left(L_{2},\|\|_{(2)}\right)$. For any $K \geqq 1$, a bijective linear map $\Phi: L_{1} \rightarrow L_{2}$ is said to be a $K$-quasi-isometry if $K^{-1}\|v\|_{(1)} \leqq\|\Phi(v)\|_{(2)} \leqq K\|v\|_{(1)}$ for all $v \in L_{1}$. By an abuse of language we shall call the quantity

$$
\begin{gathered}
\operatorname{dist}\left(\left(L_{1},\|\|_{(1)}\right),\left(L_{2},\|\|_{(2)}\right)\right)(\in[0, \infty]) \\
:=\inf \left\{\log K \mid \text { there exists a } K \text {-quasi-isometry between }\left(L_{i},\|\|_{(i)}\right)\right\}
\end{gathered}
$$

the distance between $\left(L_{1},\|\|_{(1)}\right)$ and $\left(L_{2},\|\|_{(2)}\right)$. A normed vector space $(L,\|\|)$ will be said to be $1-K$-irreducible (resp. $\infty$-K-irreducible) if $L \neq\{0\}$ and $L$ is not $K$-quasi-isometric to the 1 -direct sum (resp. the $\infty$-direct sum) of two nontrivial subspaces of $L$ equipped with the induced norm. Moreover we say that $(L,\|\|)$ is purely $1-K$-irreducible (resp. purely $\infty-K$-irreducible) if every (nontrivial) subspace of $L$ is $1-K$-irreducible (resp. $\infty$ - $K$-irreducible) in the above sense. The following is obvious from the definition. 
Proposition 2.1. If $1 \leqq \operatorname{dim} L<\infty$ and the subset $\{v \in L \mid\|v\|=1\}$ is a $C^{1}$-smooth hypersurface of $L$, then there exists a $K>1$ such that $L$ is both purely $1-K$-irreducible and purely $\infty$-K-irreducible.

For any finite Riemann surface $R$, as is well known $A_{2}^{1}(R)$ is finite dimensional and the unit sphere of $A_{2}^{1}(R)$ is $C^{1}$-smooth (cf. $[\mathrm{F}-\mathrm{K}],[\mathrm{R}],[\mathrm{E}-\mathrm{K}]$ ). Accordingly we have the following.

Proposition 2.2. For any finite Riemann surface $R$ with $A_{2}^{1}(R) \neq\{0\}$, there exists a $K>1$ such that $A_{2}^{1}(R)$ is purely $1-K$-irreducible.

COROLLARY 2.3. Under the above situation the dual space of $A_{2}^{1}(R)$ is purely $\infty$-K-irreducible for the same $K$.

For any countably many normed vector spaces $\left\{\left(L_{i},\|\|_{(i)}\right)\right\}_{i \in \mathbf{N}}$ we put

$$
\bigoplus_{i \in \mathbf{N}}^{1} L_{i}=\left\{f \in \prod_{i \in \mathbf{N}} L_{\imath} \mid\|f\|_{1}:=\sum_{i \in \mathbf{N}}\left\|f_{i}\right\|_{(i)}<\infty\right\}
$$

and

$$
\bigoplus_{i \in \mathbf{N}}^{\infty} L_{i}=\left\{f \in \prod_{i \in \mathbf{N}} L_{i} \mid\|f\|_{\infty}:=\sup _{i \in \mathbf{N}}\left\|f_{\imath}\right\|_{(i)}<\infty\right\}
$$

Here $f_{i}$ denotes the $i$-th component of $f$.

Theorem 2.4. Let $K \in(1, \sqrt[4]{2})$ and let $\left\{\left(L_{i},\|\|_{(i)}\right)\right\}_{i \in \mathbf{N}},\left\{\left(L_{i}^{\prime},\|\|_{(i)}^{\prime}\right)\right\}_{i \in \mathbf{N}}$ be two sequences of finite dimensional normed vector spaces such that $\bigoplus_{i \in \mathbf{N}}^{\infty} L_{i}$ and $\bigoplus_{i \in \mathbf{N}}^{\infty} L_{i}^{\prime}$ are mutually $K$-quasi-isometric. Suppose that $\left(L_{\imath},\|\|_{(i)}\right)$ and $\left(L_{i}^{\prime},\|\|_{(i)}^{\prime}\right)$ are all purely $\infty-K^{3}$-irreducible. Then there exists a bijective map $\tau: \mathbf{N} \rightarrow \mathbf{N}$ such that $\left(L_{\imath},\|\|_{(i)}\right)$ and $\left(L_{\tau(i)}^{\prime},\|\|_{\tau(i)}^{\prime}\right)$ are mutually $K^{2}$-quasi-isometric for all $i \in \mathbf{N}$.

Proof. For simplicity we shall suppress the subscripts for the norm. Let $\Psi$ : $\bigoplus_{i \in \mathbf{N}}^{\infty} L_{i} \rightarrow \bigoplus_{i \in \mathbf{N}}^{\infty} L_{i}^{\prime}$ be any $K$-quasi-isometry. For any $i \in \mathbf{N}$ and for any $u \in L_{i}$ satisfying $\|u\|=1$ we put

$$
\Psi(u)=\sum_{j \in \mathbf{N}} v_{j}, \quad v_{j} \in L_{j}^{\prime}
$$

Since $\Psi$ is a $K$-quasi-isometry we have $K^{-1} \leqq \sup _{j}\left\|v_{j}\right\| \leqq K$.

Let us put 


$$
\Psi^{-1}\left(v_{j}\right)=\sum_{k \in \mathbf{N}} u_{k}^{j}, \quad u_{k}^{j} \in L_{k}
$$

Clearly $\left\|u_{k}^{j}\right\| \leqq \frac{1}{2} K^{2} \leqq K^{-2}$ if $k \neq i$, so that

$$
K^{-2} \leqq \sup _{j}\left\|u_{i}^{j}\right\| \leqq K^{2}
$$

In particular there exists a $j$ such that $K^{-2} \leqq\left\|v_{j}\right\| \leqq K^{2}$ and $K^{-3} \leqq\left\|u_{i}^{j}\right\| \leqq K^{3}$. Let us take another $u^{\prime} \in L_{i}$ with $\left\|u^{\prime}\right\|=1$ and $l \in \mathbf{N}$ such that $K^{-2} \leqq\left\|v_{l}^{\prime}\right\|$ $\leqq K^{2}$ and $K^{-3} \leqq\left\|u_{i}^{\prime l}\right\| \leqq K^{3}$. Here $v_{l}^{\prime}$ and $u_{i}^{\prime l}$ are defined similarly as $v_{j}$ and $u_{k}^{j}$. Then $j$ and $l$ must be equal because $L_{\imath}$ is purely $\infty_{-} K^{3}$-irreducible.

Hence the map

$$
\begin{aligned}
\Psi_{i}: L_{i} & \rightarrow L_{j}^{\prime} \\
w & \\
u & \rightarrow v_{j}
\end{aligned}
$$

is well-defind and gives a $K^{2}$-quasi-isometry onto a subspace of $L_{j}^{\prime}$.

Interchanging and role of $L_{\imath}$ and $L_{j}^{\prime}$ we have a $K^{2}$-quasi-isometry from $L_{j}^{\prime}$ onto a subspace of $L_{i}$ for the same $i$ and $j$. Since $\operatorname{dim} L_{i}$ and $\operatorname{dim} L_{j}^{\prime}$ are finite, it follows that $\Psi_{i}$ is surjective. Thus we may put $j=\tau(i)$.

\section{§3. Non-equivalence of Teichmüller spaces}

Let $\left\{R_{i}\right\}_{i \in \mathbf{N}}$ be a sequence of finite and hyperbolic Riemann surfaces of the form $R_{1}=\hat{R}_{1} \backslash\left\{p_{1}\right\}$ and $R_{i}=\hat{R}_{i} \backslash\left\{p_{i}, q_{i-1}\right\}$ for $i \geqq 2$. Here $p_{i}$ and $q_{i-1}$ are distinct points of $\hat{R}_{i}$. We choose any local coordinates $z_{i}: D_{i} \rightarrow \Delta$ (resp. $w_{i}: E_{i} \rightarrow \Delta$ ) around $p_{i}$ (resp. $q_{i}$ ) so that $D_{i} \cap E_{i-1}=\emptyset$ for all $i \geqq 2$.

Then we put $\mathscr{R}_{i}=\left(R_{i},\left(D_{i}, z_{i}\right),\left(E_{i-1}, w_{i-1}\right)\right)$ where $\left(E_{0}, w_{0}\right):=\emptyset,\left|\mathscr{R}_{i}\right|=$ $R_{i}$ and

$$
\mathscr{R}=\left\{\mathscr{R}_{i}\right\}_{i \in \mathbf{N}} .
$$

Given any sequence $r=\left\{r_{i}\right\}_{i \in \mathbf{N}} \subset(0,1)$ we define Riemann surfaces $\mathscr{R}_{\leqq i}[r]$, inductively on $i$, by defining $\mathscr{R}_{\leqq 1}[r]=R_{1}$ and

$$
\mathscr{R}_{\leqq i+1}[r]=\left(\mathscr{R}_{\leqq i}[r], D_{i}, z_{i}\right) \#_{r_{i}}\left(R_{i+1}, E_{i}, w_{i}\right) .
$$

We then define $\mathscr{R}[r]$ as the inductive limit of $\left\{\mathscr{R}_{\geqq i}[r]\right\}_{i \in \mathbf{N}}$.

Letting $\mathscr{R}_{i, 1}=\left(\mathscr{R}_{i+1},\left(D_{i+1}, z_{\imath+1}\right), \emptyset\right), \mathscr{R}_{i, j}=\left(\mathscr{R}_{i+j},\left(D_{i+j}, z_{i+j}\right),\left(E_{i+j}, w_{i+j}\right)\right)$ for $j \geqq 2$, 


$$
\mathscr{R}_{>i}=\left\{\mathscr{R}_{1, j}\right\}_{j \in \mathbf{N}}
$$

and $r_{>i}=\left\{r_{i+j}\right\}_{j \in \mathbf{N}}$ we put $\mathscr{R}_{>i}[r]=\mathscr{R}_{>i}\left[r_{>i}\right]$.

Note that

$$
\mathscr{R}[r]=\left(\mathscr{R}_{\leqq i}[r], D_{i}, z_{i}\right) \#_{r_{i}}\left(R_{>i}[r], E_{i}, w_{i}\right) .
$$

We shall call $\mathscr{R}$ a decorated sequence of Riemann surfaces and $\mathscr{R}[r]$ the $r$-connected sum of $\mathscr{R}$. $\mathscr{R}_{i}$ shall be referred to as the $i$-th component of $\mathscr{R}$.

Once for all we shall fix $\mathscr{R}$ and $r$. Then, for any Riemann surface $R^{\prime}$ which is $K$-quasiconformally equivalent to $\mathscr{R}[r]$, one can find a decorated sequence $\mathscr{R}^{\prime}=$ $\left\{R_{i}^{\prime}\right\}_{i \in \mathbf{N}}$ and a sequence $r^{\prime}=\left\{r_{i}^{\prime}\right\}_{i \in \mathbf{N}} \subset(0,1)$ satisfying the following properties.

$$
\mathscr{R}^{\prime}\left[r^{\prime}\right] \text { is conformally equivalent to } R^{\prime} \text {. }
$$$$
\left|\mathscr{R}_{i}^{\prime}\right| \text { is } K \text {-quasiconformally equivalent to }\left|\mathscr{R}_{i}\right| \text { for every } i \text {. }
$$

$$
r_{i}^{K} \leqq r_{i}^{\prime} \leqq r_{i}^{K^{-1}}
$$

In fact, this is a consequence of two obvious facts that the quantity $m_{r}:=-\log r$ attached to $\Delta(r, 1)$ satisfies

$$
K^{-1} m_{r_{2}} \leqq m_{r_{1}} \leqq K m_{r_{2}}
$$

if $\Delta\left(r_{1}, 1\right)$ and $\Delta\left(r_{2}, 1\right)$ are $K$-quasiconformally equivalent to each other, and that any $K$-quasiconformal homeomorphism from $\Delta\left(r_{1}, 1\right)$ to $\Delta\left(r_{2}, 1\right)$ is extendable to a $K$-quasiconformal automorphism of $\mathbf{C} \backslash\{0\}$ by iteration of the reflections along the circles centered at 0 .

THEOREM 3.1. Let $R$ be any decorated sequence of finite and hyperbolic Riemann surfaces. Then there exist two sequences $r=\left\{r_{i}\right\}, \varepsilon=\left\{\varepsilon_{i}\right\} \subset(0,1)$ with $\lim _{i \rightarrow \infty}$ $\left(r_{i}+\varepsilon_{i}\right)=0$ such that, for any quasiconformal deformation $\left(\mathscr{R}^{\prime}, r^{\prime}\right)$ of $(R, r)$ one can find a $j \in \mathbf{N}$ such that for all $k \in \mathbf{N}, A_{2}^{1}\left(\mathscr{R}_{\leq j+k}^{\prime}\left[r^{\prime}\right]\right)$ are purely $1-\left(1+\varepsilon_{j+k}\right)^{3}-$ irreducible and $A_{2}^{1}\left(\mathscr{R}^{\prime}\left[r^{\prime}\right]\right)$ (resp. $\left.A_{2}^{1}\left(\mathscr{R}_{>j+k}^{\prime}\left[r^{\prime}\right]\right)\right)$ is $\left(1+\varepsilon_{j+k}\right)$-quasi-isometric to $A_{2}^{1}\left(\mathscr{R}_{\leqq j+k}^{\prime}\left[r^{\prime}\right]\right) \oplus A_{2}^{1}\left(\mathscr{R}_{>j+k}^{\prime}\left[r^{\prime}\right]\right) \oplus \mathbf{C}$ (resp. $\left.\left(\oplus^{1} A_{2}^{1}\left(R_{j+k+m}^{\prime}\right)\right) \oplus l_{\mathbf{C}}^{1}\right)$. Here $l_{\mathbf{C}}^{1}$ denotes the space of absolutely summable sequence with values in $\mathbf{C}$.

Proof. The assertion is a routine consequence of Theorem 1.5 and Proposition 2.2. The detail is left to the reader.

Let $\mathscr{R}$ be as above and let $\mathscr{R}_{i}$ be its $i$-th component. If the Riemann surfaces $\left|\mathscr{R}_{\imath}\right|, i \in \mathbf{N}$, consist of only finitely many conformal equivalence classes, then we 
can choose $r$ and $\varepsilon$ independently of the order of $\mathscr{R}_{i}(i \geqq 2)$, i.e., letting $\mathscr{R}_{\tau}:=$ $\left\{\mathscr{R}_{\tau(i)}\right\}_{i \in \mathbf{N}}$ for any bijective map $\tau: \mathbf{N} \rightarrow \mathbf{N}$ satisfying $\tau(1)=1$, they can be required to satisfy the same condition as above for all $\mathscr{R}_{\tau}$. Moreover, in this situation, we can require that all the components $A_{2}^{1}\left(R_{j+k+m}^{\prime}\right)$ are purely $1-(1+$ $\left.\varepsilon_{j+k}\right)^{3}$-irreducible. The following is then deduced from Theorem 2.1.

PROPOSITION 3.2. Under the above situation, $\mathscr{R}[r]$ and $\mathscr{R}_{\tau}[r]$ are not quasiconformally equivalent if two sequences $\left\{\operatorname{dim} A_{2}^{1}\left(\left|\mathscr{R}_{i}\right|\right)\right\}_{i \in \mathbf{N}}$ and $\left\{\operatorname{dim} A_{2}^{1}\left(\left|\mathscr{R}_{\tau(i)}\right|\right)\right\}_{i \in \mathbf{N}}$ are cofinally non-equivalent.

Let $\mathscr{T}(\mathscr{R}[r])$ denote the Teichmüller space of $\mathscr{R}[r]$. In virtue of Gardiner's theorem, the tangent space of $\mathscr{T}(\mathscr{R}[r])$ at the point $[(\mathscr{R}[r], \mathrm{id})] \in \mathscr{T}(\mathscr{R}[r])$ is isometric to $A_{2}^{1}(R[r])^{*}$ with respect to the Teichmüller infinitesimal form (cf. [G, Theorem 3.5]). It is also known from the work of Gardiner that any biholomorphism between Teichmüller spaces is an isometry with respect to the Teichmüller metric (cf. [G, Corollary 2.1]). Hence we have also the following consequence of Theorem 3.1.

Proposition 3.3. Under the above situation, $\mathscr{T}(\mathscr{R}[r])$ and $\mathscr{T}\left(\mathscr{R}_{\tau}[r]\right)$ are not biholomorphically equivalent whenever $\left\{\operatorname{dim} A_{2}^{1}\left(\left|\mathscr{R}_{i}\right|\right)\right\}$ and $\left\{\operatorname{dim} A_{2}^{1}\left(\left|\mathscr{R}_{\tau(\imath)}\right|\right)\right\}$ are cofinally non-equivalent.

As an immediate corollary to Proposition 3.3 we obtain the following.

THEOREM 3.4. There exist uncountably many biholomorphically non-equivalent Teichmüller spaces.

Furthermore, if $r$ and $\varepsilon$ are sufficiently rapidly decreasing, then the $\left(1+\varepsilon_{j}\right)^{2}$-quasi-isometry between $A_{2}^{1}\left(\mathscr{R}_{\leqq j}^{\prime}[r]\right)$ and $A_{2}^{1}\left(\mathscr{R}_{\leqq j}^{\prime}\left[r^{\prime}\right]\right)$ will imply $K_{j}$-quasiconformal equivalence between $\mathscr{R}_{\leqq j}[r]$ and $\mathscr{R}_{\unlhd_{j}}^{\prime}\left[r^{\prime}\right]$ with $\lim _{j \rightarrow \infty} K_{j}=1$. Therefore we see that our $r$ can be chosen so that the existence of a biholomorphic automorphism $\sigma$ of $\mathscr{T}(\mathscr{R}[r])$ with $\sigma([(\mathscr{R}[r], f)])=\left[\left(\mathscr{R}^{\prime}\left[r^{\prime}\right], g\right)\right]$ forces $\mathscr{R}[r]$ and $\mathscr{R}^{\prime}\left[r^{\prime}\right]$ to be are conformally equivalent.

Under the above situation it is clear that, for any point $p \in \mathscr{T}(\mathscr{R}[r])$ one can find a locally closed submanifold $\mathscr{T}^{\prime} \subset \mathscr{T}(\mathscr{R}[r])$ of arbitrarily large dimension passing through $p$, and a neighbourhood $U \ni p$, such that every biholomorphic automorphism $\sigma$ of $\mathscr{T}(\mathscr{R}[r])$ satisfies $\sigma(p) \notin \mathscr{T}^{\prime} \backslash U$. 
According to the work of Earle and Gardiner $[\mathrm{E}-\mathrm{G}]$ it is known that any biholomorphic automorphism of the Teichmüller space of a topologically finite Riemann surface with non-empty border is induced from a quasiconformal homeomorphism between conformally equivalent surfaces. Combining this fact with the above observation we obtain

PROPOSITION 3.5. Under the above assumptions on $\mathscr{R}$ and $r, \mathscr{T}(\mathscr{R}[r])$ is not biholomorphically equivalent to the Teichmüller space of any topologically finite Riemann surface.

\section{REFERENCES}

[A-V] Andreotti, A. and Vesentini, E., Sopra un teorema di Kodaira, Ann. Scuola Norm. Sup. Pisa, (3) $\mathbf{1 5}$ (1961), 283-309.

[D] Demailly, J.-P., Estimations $L^{2}$ pour l'opérateur $\bar{\partial}$ d'un fibré vectoriel holomorphe au-dessus d'une variété kählerienne complète, Ann. Sci. Ecole Norm. Sup., 15 (1982), 457-511.

[E-G] Earle, C. J. and Gardiner, F. P., Geometric isomorphisms between infinite dimensional Teichmüller spaces, preprint.

[E-K] Earle, C. J. and Kra, I., On holomorphic mapping between Teichmüller spaces, Contribution to analysis (a collection of papers dedicated to Lipman Bers) pp. 107-124. Academic Press, New York, 1974.

[E-K-K] Earle, C. J., Kra, I. and Krushkal', S. L., Holomorphic motions and Teichmüller spaces, Trans. Amer. Math. Soc., 343 (1994), 927-948.

[F-K] Farkas, H. and Kra, I., Riemann surfaces (second edition) GTM 71, Springer 1991, New York Berlin Heidelberg.

[G] Gardiner, F., Approximation of infinite dimensional Teichmüller spaces, Trans. Amer. Math. Soc., 282 (1984), 367-383.

[Kru] Krushkal', S. L., Strengthening pseudoconvexity of finite-dimensional Teichmüller spaces, Math. Ann., 290 (1991), 681-687.

[N] Nag, S., A period mapping in universal Teichmüller space, Bull. Amer. Math. Soc., 26 (1992), 280-287.

[O-1] Ohsawa, T., On complete Kähler manifolds with $C^{1}$-boundary, Publ. RIMS, Kyoto Univ., 16 (1980), 929-940.

[O-2] - Vanishing theorems on complete Kähler manifolds, Publ. RIMS, Kyoto Univ., 20 (1984) 21-38.

[Ri] Richberg, R., Stetige streng pseudokonvexe Funktionen, Math. Ann., 175 (1968), $257-286$.

[R] Royden, H., Automorphisms and isometries of Teichmüller space, Advances in the theory of Riemann surfaces (Proc. Conf. Stony Brook, N. Y., 1969) pp. 369-383 Ann. Math. Stud., 66 Princeton Univ. Press, Princeton, N. J., 1971.

[T] Tanigawa, H., Holomorphic families of geodesic discs in infinite dimensional Teichmüller spaces, Nagoya Math. J., 127 (1992), 117-128. 
Graduate School of Polymathematics

Nagoya University

Chikusa-ku, Nagoya 464-01

Japan 